\title{
Putting on the brakes: NOAC antidotes
}

Although the non-vitamin $\mathrm{K}$ antagonist oral anticoagulants (NOACs) improved patient outcomes in clinical trials (MILESTONE 10), they entered the market without an antidote. Perhaps owing to the clinical experience with vitamin $\mathrm{K}$ antagonists (MILESTONE 2), the effects of which can be reversed by treatment with prothrombin complex concentrate or vitamin $\mathrm{K}$, clinicians and patients were concerned about the absence of a method to reverse anticoagulation if patients experience major bleeding or trauma, or require emergency surgery. Indeed, one of the adverse effects of NOACs is major bleeding, which occurs in $\sim 2-3 \%$ of patients taking these drugs.

Three NOAC antidotes have been investigated: idarucizumab (Praxbind, approved by the FDA); andexanet alfa (andexanet, Biologics License Application under FDA review), and ciraparantag (in phase II trials).

Idarucizumab, a monoclonal antibody fragment that binds dabigatran, received FDA approval in 2015. The pivotal phase III trial was a multicentre, prospective, open-label study that involved 503 patients with uncontrolled bleeding (group A, 301 patients) or who were about to undergo an urgent procedure (group B, 202 patients).
Patients in group A predominantly presented with gastrointestinal bleeding or intracranial haemorrhage, and the antidote stopped bleeding in a median time of $2.5 \mathrm{~h}$. For patients in group B, haemostasis was achieved before surgery (median $1.6 \mathrm{~h}$ after idarucizumab administration) in $93.4 \%$ of patients.

\section{Antidotes could substantially improve the safety of NOACs}

Andexanet is a recombinant modified human factor Xa protein that is catalytically inactive, but can bind to factor Xa inhibitors, and thus acts as a decoy. Whereas idarucizumab can be used only to reverse the anticoagulant activity of dabigatran, andexanet is a potential antidote for any of the approved direct factor Xa inhibitors (apixaban, betrixaban, edoxaban, or rivaroxaban). In healthy volunteers aged 50-75 years who were given apixaban or rivaroxaban, thrombin generation was fully restored within $2-5 \mathrm{~min}$ of andexanet administration in $100 \%$ of patients. In a multicentre, prospective,

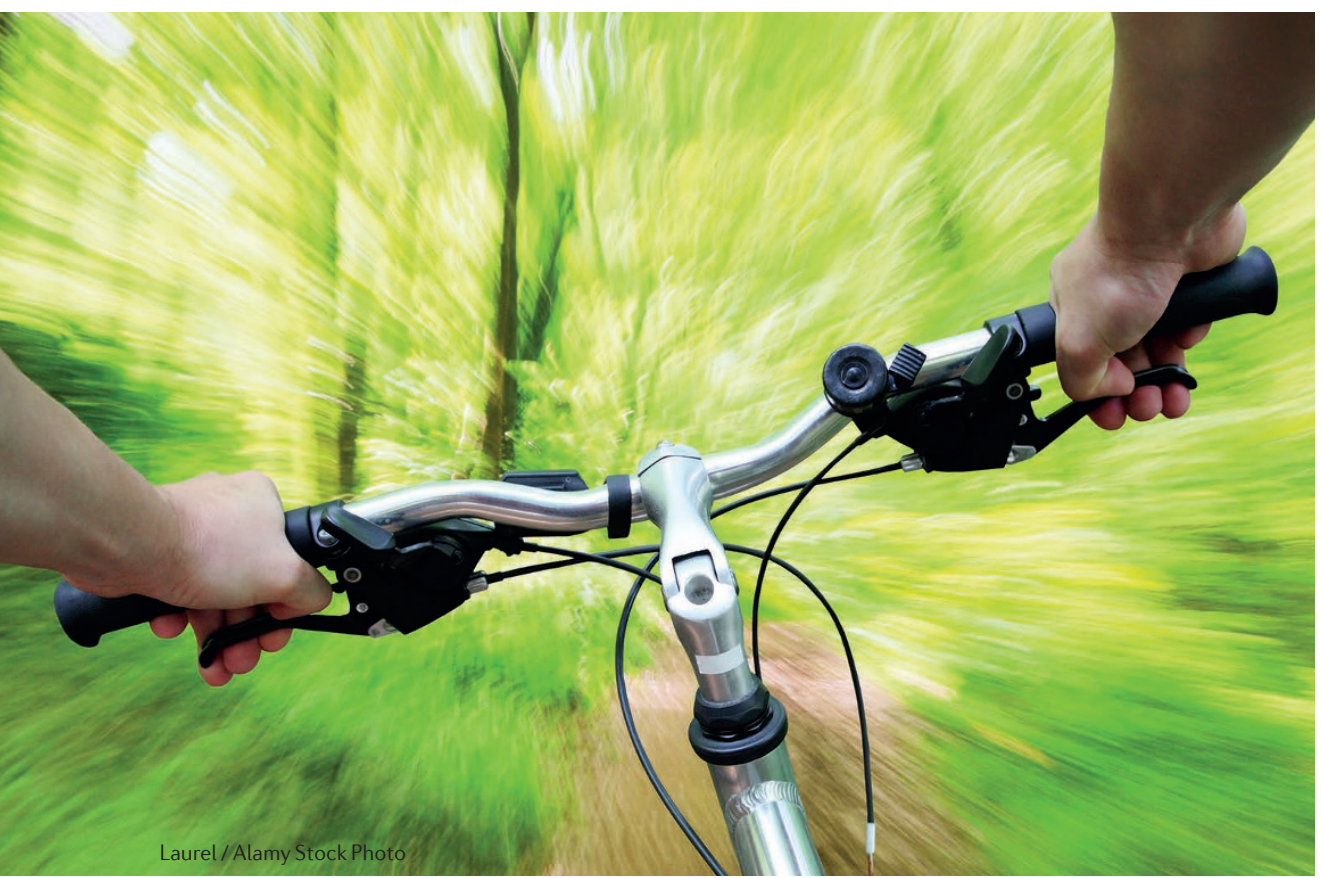

open-label, single-group study, 67 patients who had acute major bleeding within $18 \mathrm{~h}$ after administration of a factor Xa inhibitor were treated with andexanet (bolus, followed by a 2 -h infusion). Treatment reduced factor Xa activity by $89 \%$ and $93 \%$ in patients who were taking rivaroxaban or apixaban, respectively.

Ciraparantag is a synthetic small molecule. This compound binds and inhibits the direct factor Xa inhibitors as well as dabigatran and heparin, so could provide broader anticoagulation reversal than either idarucizumab or andexanet if proven to be effective.

Achieving the right balance between promoting and preventing coagulation is challenging, and thrombotic events were observed in the 30-day or 90-day follow-up period in the trials for andexanet or idarucizumab, respectively. Both of these trials were conducted in populations of elderly patients with substantial comorbidities and serious index events, many of whom did not reinitiate anticoagulant treatment for underlying conditions following antidote administration. The contribution of the antidote to these adverse events is not clear, and most of the events occurred after the antidotes would have been metabolized and excreted, but the antidotes might have induced longer-lasting effects.

Idarucizumab was approved under the FDA's accelerated approval programme, and andexanet is under consideration as a breakthrough therapy - designations granted to treatments for life-threatening conditions or that fulfil major unmet medical needs. Both of these designations require the collection of post-marketing data on safety and efficacy. A 2015 meeting of the cardiac safety research consortium, a public-private partnership aimed to support research into the evaluation of cardiac safety of medical products, strongly supported the collection of phase IV data as an essential part of the approval process.

Antidotes could substantially improve the safety of NOACs, providing a mechanism to 'put the brakes' on anticoagulation. Continued data collection as these therapies enter the clinic will determine the extent to which they can improve patient outcomes.

Megan Cully,

Senior Editor, Nature Reviews Drug Discovery

ORIGINAL ARTICLES Pollack, C. V. et al. Idarucizumab for dabigatran reversal - full cohort analysis. N. Engl.J. Med. 377 , 431-441 (2017) | Siegal, D. M. et al. Andexanet alfa for the reversal of factor Xa inhibitor activity. N. Engl.J. Med. 373 2413-2424 (2015) | Connolly, S. J. et al. Andexanet alfa for acute major bleeding associated with factor Xa inhibitors. N. Engl.J. Med. 375, 1131-1141 (2016) FURTHER READING Reiffel, J. A. et al. NOAC monitoring, reversal agents, and post-approval safety and effectiveness evaluation: a cardiac safety research consortium think tank. Am. Heart J. 177, 74-86 (2016) 